\title{
SUBSTRUCTURE AND LOCAL ORIENTATION EVOLUTION OF ALUMINUM SINGLE CRYSTALS DURING DEFORMATION
}

\author{
H. Weiland*, J. Liu ${ }^{*}$ and E. Nes ${ }^{+}$ \\ * Alcoa Laboratories, Alcoa Center, PA 15069, USA \\ + Norwegian Institute of Technology, Dep. of Metallurgy, N-7034 Trondheim, \\ Norway
}

\section{INTRODUCTION}

The evolution of the crystallographic substructure in deformed aluminum single crystals has been analyzed by measuring misorientations between subgrains using convergent beam electron diffraction (CBED). The variation of the subgrain size is mostly used in characterizing the evolution of a substructure during deformation 1 . This gives some information about the stored energy in the material during the deformation process. The stored energy not only depends on the subgrain size, but also on the subboundary energy, which is a function of the misorientation. Some earlier work has shown that the subboundary misorientation varies with the strain 2,3 . But the orientation difference across the subboundary has not been the subject of extensive investigations, because techniques capable of determining a sufficient number of misorientations with an accuracy better than one degree in an acceptable amount of time were missing. Recent developments in electron microscopic techniques overcome this problem and now provide fast single orientation measurement techniques using Kikuchi patterns in transmission (CBKP) 4 or in reflection (EBSP) 5 .

The appearance of systematical changes in misorientation over long ranges is investigated here by determining misorientations between the orientations of the grains of interest with respect to the orientation of one fixed grain. This requires a representation of misorientations as a function of the spacing which is a six-dimensional problem. Therefore new approaches for the analysis of long-range misorientations are developed, such as a new way of making use of Rodrigues vectors and the definition of a misorientation correlation index.

\section{EXPERIMENTAL PROCEDURE}

Single crystals of high purity aluminum with the orientations (112)[111] and (001)[110] were channel die deformed to strains of 0.2 and 1.0 (for details see ref. 6). The first orientation is known to be stable and the second one to be unstable with respect to plane strain deformation. The substructure consists of small, elongated subgrains. The subgrain size varied from 1.5 to $4.5 \mu \mathrm{m}$ in the low deformed condition and from 0.15 to $2.5 \mu \mathrm{m}$ in the high deformed one. For a more detailed discussion and for micrographs of the microstructure see ref. 7 . 
Single orientations of neighboring subgrains were determined in a TEM by the online measurement of Kikuchi patterns obtained from CBED4. The TEM foils were prepared from the longitudinal planes. Taking advantage of the fact that Kikuchi patterns can be obtained until the limit of transmission in thin specimen, the measurements were done in thicker specimen areas in order to avoid the influence of a possible bending of the TEM foil. Furthermore, the measurements were carried out with the highest possible accuracy, so that the theoretical precision of 0.1 degree with respect to neighboring crystal coordinate systems in the orientation determination was achieved. Orientations of neighboring subgrains were measured over distances of $120 \mu \mathrm{m}$ in the extension (ED) as as well in the compression direction (CD). At the high strain level, this included ca. 240 orientations in $\mathrm{CD}$ and ca. 140 orientations in ED and for the lower strain level 100 (CD) / 70 (ED) orientations.

\section{DESCRIPTION OF LONG-RANGE MISORIENTATIONS}

A misorientation is often described by the rotation axis/ -angle, which transforms one crystal coordinate system into another crystal coordinate system one around a common rotation axis. For crystals with cubic symmetry, the misorientation between two grains can be described by 24 such axis-angle pairs. Of these the one with the minimum misorientation angle alone has been used most frequently. The description of the corresponding rotation axis requires a separate representation such as a pole figure. Alternatively, a complete description of misorientations can be obtained using Rodrigues vectors 8 . The direction of the vector in the Rodrigues space characterizes the rotation axis and its length characterizes the rotation angle. In order to link the misorientation space with the real space, the origin of the Rodrigues space for each subsequent misorientation can be positioned at the real-space coordinates of the center of gravity of the corresponding (sub-) grain or at the coordinates of the coordinates of the corresponding grain boundary. In this way a description of the six-dimensional space (the misorientation space and the real space) can be achieved 9 . To be more precise, the 3D orientation space is superimposed on the 3D real space. Due to the orthotropic geometry of plane strain deformation, the spatial coordinates of the individual orientation measurement conveniently can be chosen to coincide with the three orthogonal sample axes. The Rodrigues vectors describing the misorientation with respect to the orientation of a reference grain can be plotted along

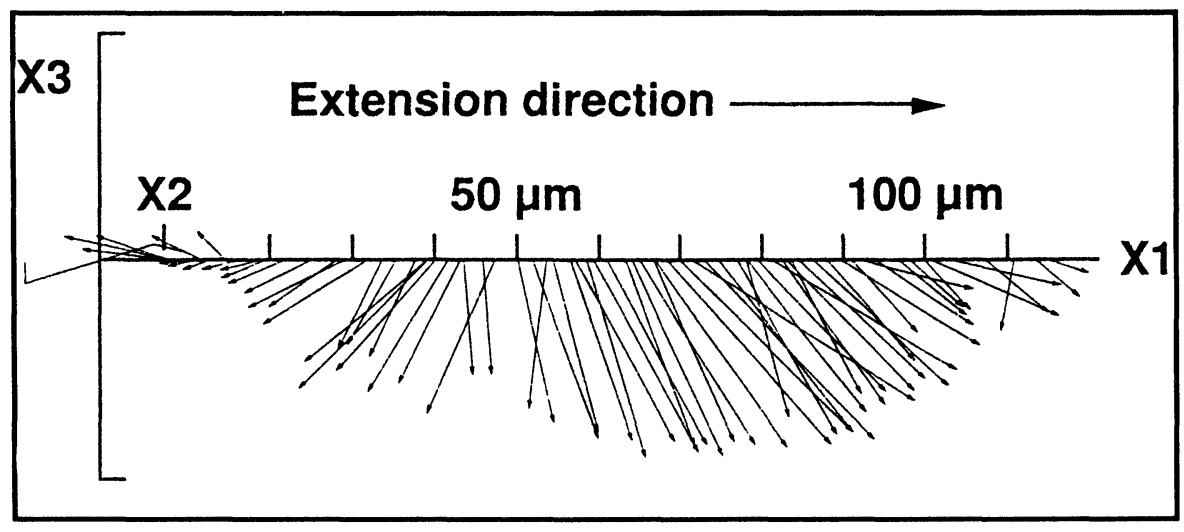

Figure 1. Perspective representation of misorientations as Rodrigues vectors along a specimen direction (stable orientation deformed to a strain of 0.2 ). X1,X2,X3 are the coordinates of the Rodrigues space; XI and the extension direction are parallel. 
one specimen direction at those coordinates, where the corresponding subgrains / grainboundaries are situated in the microstructure. If the misorientation of each subgrain is expressed with respect to a reference grain, long-range misorientations can be described along a direction of interest.

For example, the misorientations with respect to a reference grain positioned at the origin of the spatial axis are plotted in figure 1. The data are from the crystal with the stable orientation deformed to a strain of $\mathbf{0 . 2}$. The rotation axis, corresponding to the direction of the Rodrigues vector in space, as well as the rotation angle (length of the Rodrigues vector) vary systematically along the extension direction of the deformed crystal. For the sake of simplifying, the vectors should be regarded here only with respect to the X3 direction. Beginning from $+\mathrm{X} 3$ the Rodrigues vectors rotate towards -X3 with increasing distance and then, at a distance of $55 \mu \mathrm{m}$, the vectors start to rotate back towards the initial position. This figure clearly shows that long-range misorientations are present in the deformed material. A representation of misorientations only by the rotation angles would not be sufficient.

The description of misorientations by Rodrigues vectors as given above strongly depends on the choice of the reference grain/-orientation. As no grain and therefore no orientation is predetermined in a microstructure, the choice of the reference grain is arbitrary. While the Rodrigues space is very useful in visualizing long-range misorientations, a deterministic solution for the described effects is needed.

Misorientations can also be described by Euler angles. Misorientation distribution functions (MODFs) 10 are then obtained by applying the series expansion theory. In a way similar to the texture index, which is used in characterizing orientation distribution functions (ODFs), a Misorientation Correlation Index (MCI) can be defined 11. This index is then the summation of the appropriate indices $\mathrm{D}^{\mu \nu}$ of the series expansion:

$$
\mathrm{MCI}=\sum_{l=0}^{L_{\text {max }}} \sum_{\mu=1}^{\mathrm{M}(l)} \sum_{v=1}^{\mathrm{N}(l)} \frac{1}{21+1}\left[\mathrm{D}_{1}^{\mu \nu}\right]^{2}
$$

For a detailed explanation of the parameters in equation 1 as well as the application of the series expansion method for misorientation distributions see ref. 10.

This expression will characterize the MODF of the $n^{\text {th }}$ neighboring subgrains, e.g. the MODF of the next neighbors. The MCI can be obtained from MODF's of (sub-) grains which are 1st, 2nd, 3rd neighbors, and so on. Thus, each distribution is characterized by one parameter. The variation of the MCI with (sub-) grain distances links spatial coordinates and orientations in a substructure (see for example figure 2). The boundary conditions of the MCI are fixed. At a subgrain distance of zero, which is the grain itself, the MCI is completely defined by the symmetrically invariant functions $\ddot{\mathrm{T}}_{1}^{\mu \nu}$ at $\varphi_{1}=\Phi=\varphi_{2}=0^{\circ}$ and by the degree of the series expansion. Therefore, the MCI can be normalized to one at this point. When no correlation can be expected, as at large subgrain distances, the MODF is completely determined by the ODF. This so-called uncorrelated MODF can be obtained by the convolution integral of the ODF 10 under the assumption that all orientations of a texture are randomly distributed in space. The correlation index of the uncorrelated MODF is then the limiting value, which the MCI for large subgrain distances should approach. So the MCI equals one for a high correlation and reaches zero for the uncorrelated case. 


\section{RESULTS AND DISCUSSION}

Figure 2 shows the MCI for the crystal with the initial orientation (112)[111] channel die deformed to a strain of 0.2 . The MCI was determined for subgrain distances up to 50 in increments of one in two specimen directions (ED/CD). The correlation index for the random case was obtained from the convolution integral of the ODF.

The MCI along the extension direction shows evidence of a periodicity with a wave length of 13 subgrains. This means that the correlation between grains which have a spatial distance to each other of 13 subgrains actually becomes higher than many subgrains that are closer together. This periodicity of the MCI is not accompanied by any morphological periodicity (see figure 1a in ref. 7). The MCI is still rather high even at 50 subgrains away, suggesting a reasonably strong correlation at such a large distance. However, along the compression direction, the periodicity is much less evident, and at a distance of 50 subgrains, the MCI decreases to approach the limiting value determined from the ODF.

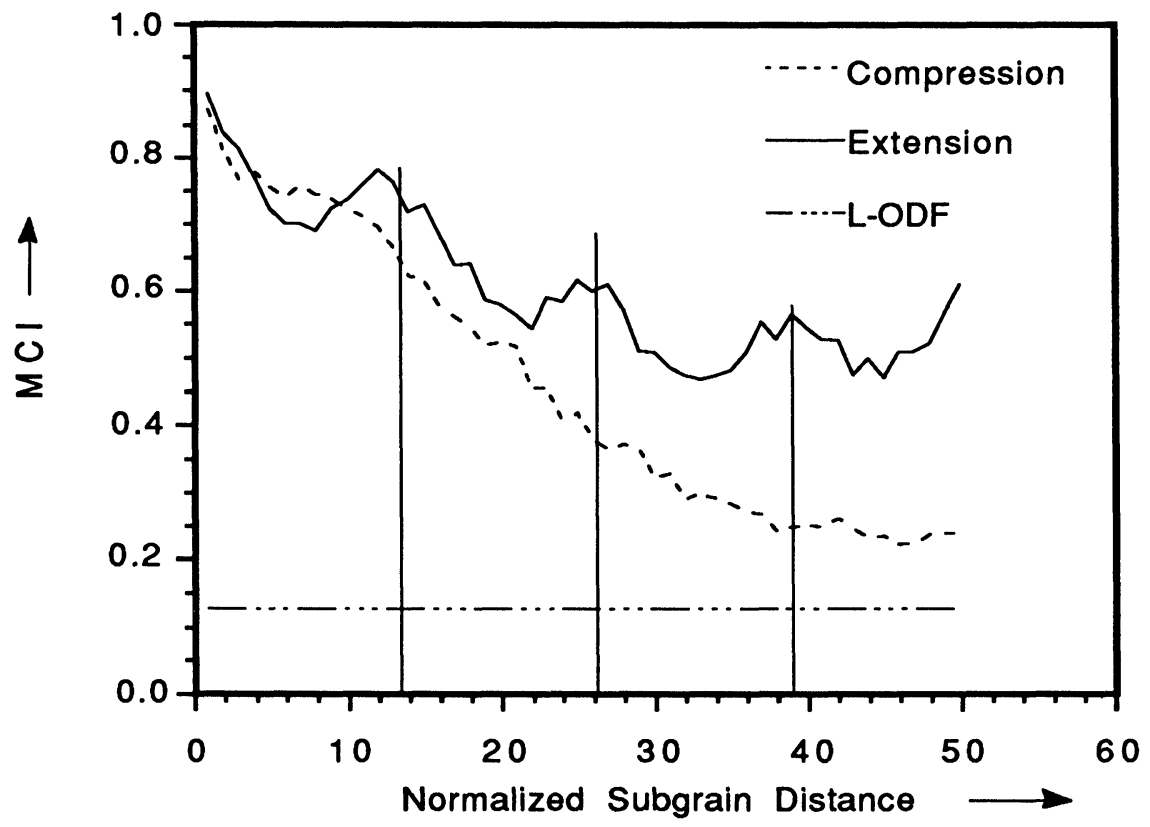

Figure 2 Misorientation Correlation Index as a function of normalized subgrain distance in a channel die deformed Al single Crystal of the orientation (112) $[11 \overline{1} \mid$ at a strain of 0.2.L-ODF is the correlation index obtained from the convolution integral of the $O D F$.

At the higher strain $(\varepsilon=1.0)$, the misorientation correlation index decreases rapidly within a distance of approximately five to seven subgrains for both specimen directions. The periodicity of the MCI cannot be resolved any further (figure 3). On the other hand bands are now visible in the microstructure, which are aligned parallel to the traces of the secondary slip system (figure $1 \mathrm{~b}$ in ref. 7). These bands show the same periodicity as the 
MCI had at a strain of 0.2 . It appears that they have been triggered by the perturbations present in the misorientations at $\varepsilon=0.2$.

The unstable orientation does not reveal a distinct subgrain formation at the low strain. Therefore, no misorientation patterns are developed in this condition. At high strains, however, most crystals have rotated towards both variants of the stable orientation. By this means transition bands are formed. It should be mentioned that in transition bands no smooth orientation changes were observed between both final orientations, and therefore no orientations according to a Dillamore-Katoh 12 mechanism were generated. The changes are rather abrupt with orientation differences in the range of $60^{\circ}$ (see also ref. 7). The corresponding MCI (figure 4) shows in principel the same pattern as occurred for the stable orientation. Again the MCI tends to decrease rapidly within a distance of five to seven neighbors and then its curve flattens out.

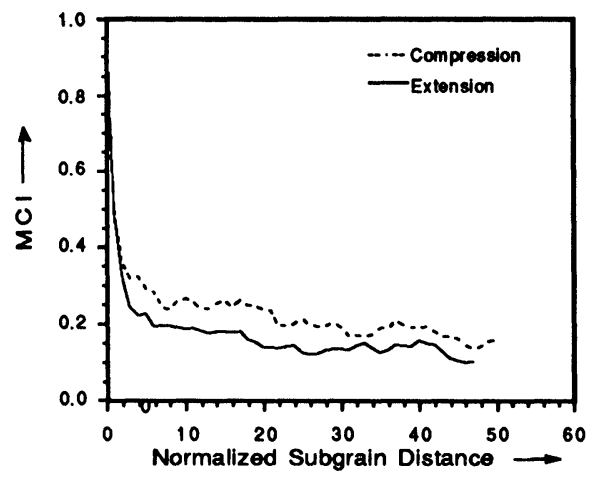

Figure 3 Misorientation Correlation Index as a function of normalized subgrain distance in an Al single crystal of the initial orientation (112)[11-1], channel die deformed to a strain of 1.0

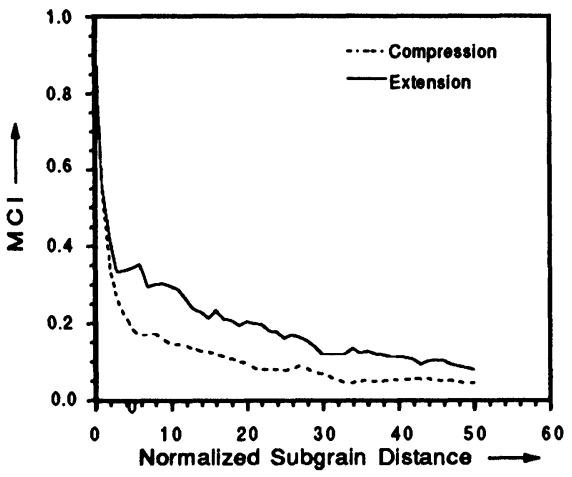

Figure 4. Misorientation Correlation Index as a function of normalized subgrain distance in an $\mathrm{Al}$ single crystal of the initial orientation (001) [100], channel die deformed to a strain of 1.0

\section{CONCLUSION}

The application of the misorientation correlation index to the evolution of long-range misorientations in the analyzed single crystals has the following results:

The rapid decrease of the MCI in both crystals at a strain of 1.0 is in good agreement with the analysis of Hjelen et al. ${ }^{7}$. These researchers used a different approach for describing long-range misorientations and used SEM techniques for the measurement of individual orientations in studying accumulative misorientations in the same single crystals as well as in polycrystalline aluminum. They showed that an accumulation of misorientations exists on a local scale up to the fifth to seventh neighbor, which is in excellent agreement with the findings in this paper.

The local orientation and substructure evolution during deformation of a single crystal with the initial orientation (112)[11 1 ] can be described as follows (Table 1). In an ideal undeformed single crystal no substructure is initially present. With beginning deformation, dislocations, which are scattered in the matrix chaotically, are generated. In the next step, subgrains are formed in the microstructure. The cells are elongated parallel to the primarily most active slip system. At the same time, the strains are accommodated 
by the development of orientational inhomogeneities manifested by the wavy pattern observed in the long-range misorientations. After a further increase of the strain, these orientational inhomogeneities are relaxed, forming a band structure which is extended in the direction of the secondary most active slip system. Further increasing of the strain will result in a substructure with subgrain boundaries aligned parallel to the deformation direction.

We think that this local orientation and substructure evolution is not only characteristic for the (112)[11 $\overline{1}]$ orientation;but it is in fact a general material behavior.

Table 1 Local orientation and substructure evolution during deformation of an Al single crystal with the initial orientation (112)[11-1]

\begin{tabular}{cll}
$\begin{array}{l}\text { Stage of } \\
\text { deformation }\end{array}$ & Orientation & Substructure \\
\hline No strain & single crystal & - \\
$1^{\text {st }}$ stage & primary slip & scattered dislocations \\
$2^{\text {nd }}$ stage & $\begin{array}{l}\text { development of } \\
\text { orientational inhomogeneities }\end{array}$ & $\begin{array}{l}\text { subgrain formation } \\
\text { [cells elongated parallel } \\
\text { to primary slip traces] }\end{array}$ \\
$3^{\text {rd }}$ stage & $\begin{array}{l}\text { secondary slip } \\
\text { orientational inhomogeneities } \\
\text { relaxed }\end{array}$ & $\begin{array}{l}\text { band formation } \\
\text { [parallel to secondary slip } \\
\text { traces] }\end{array}$
\end{tabular}

\section{ACKNOWLEDGEMENT}

The authors wish to thank J.F. Butler, University of Pittsburgh, for the preparation of the single crystals.

\section{REFERENCES}

1. J. Gil Sevillano, P. Van Houtte and E. Aernoudt, Proc. Material Sci, 25, 69 (1980)

2. F. Schuh and M. von Heimendahl, Z. Metallkunde, 65, 396 (1980)

3. E. Nes, A.L. Donas and N. Ryum, Proc. ICSMA 6, 425-430, (1982)

4. R. Schwarzer and H. Weiland, Proc. ICOTOM 7, 839-843 (1984)

5. D. J. Dingley, Scanning Electron Microscopy, 1984/II, pp. 569-575

6. J. F. Butler, Jr., Ph. D. Thesis, University of Pittsburgh (1990)

7. J. Hjelen, H. Weiland, J. Butler, J. Liu, H. Hu and E. Nes, this conference

8. R. Becker and S. Panchanadeeswaran, Texture and Microstructures, 10, (1989), pp. 167-194

9. H. Weiland, to be published in Textures and Microstructures

10. H. J. Bunge and H. Weiland, Textures and Microstructures, 7 (1988), pp. 231-263

11. H. Weiland, submitted to Acta Met.

12. I.L. Dillamore and H. Katoh, Metal Science Vol. 8 (1974) 73-83. 